\title{
8 \\ The HPA Axis and Functional Somatic Symptoms
}

\begin{abstract}
In this chapter we continue our exploration of the neurobiology of functional somatic symptoms by considering the role of the hypothalamic-pituitary-adrenal (HPA) axis. It is a complicated system that helps to regulate energy, and that affects cell function and gene expression, throughout the body. In addition to playing a fundamental role in body regulation day to day, the HPA axis activates in response to stress and, when dysregulated (via either chronic hyper- or hypo-activation), plays a central role in all stress-related disorders. The vignettes in the chapter are designed to bring the neurobiology and dysregulation of the HPA axis, which are often difficult to detect, more into clinical focus.
\end{abstract}

As with all life processes, the body's efforts to regulate and maintain its internal environment-its milieu intérieur (see Online Supplement 1.2)—require energy. More broadly, all of the body's efforts to adapt to

Electronic supplementary material The online version of this chapter (https://doi.org/10.1007/978-3-030-46184-3_8) contains supplementary material, which is available to authorized users.

(C) The Author(s) 2020

K. Kozlowska et al., Functional Somatic Symptoms in Children and Adolescents, Palgrave Texts in Counselling and Psychotherapy, https://doi.org/10.1007/978-3-030-46184-3_8 
the everyday challenges, stress, and dangers of daily life require energy, and it is the hypothalamic-pituitary-adrenal (HPA) axis that makes the moment-by-moment adjustments required for that purpose.

The HPA axis is part of the body's energy-regulation system-a coordinated system that spans multiple system levels, from brain systems that continually anticipate the body's energy needs (Kleckner et al. 2017) to energy-regulation mechanisms in each individual cell (Picard et al. 2018). Any activation of the stress system in response to physical or psychological stress involves concomitant adjustments in energy regulation. The HPA axis relies on hormones for communication and signalling; these protein molecules serve 'as messengers between cells, telling them what's happening elsewhere and how they should respond' (Kushiro et al. 2003, p. 203) (see Fig. 8.1). This sort of chemical-communication system is thought to have originated

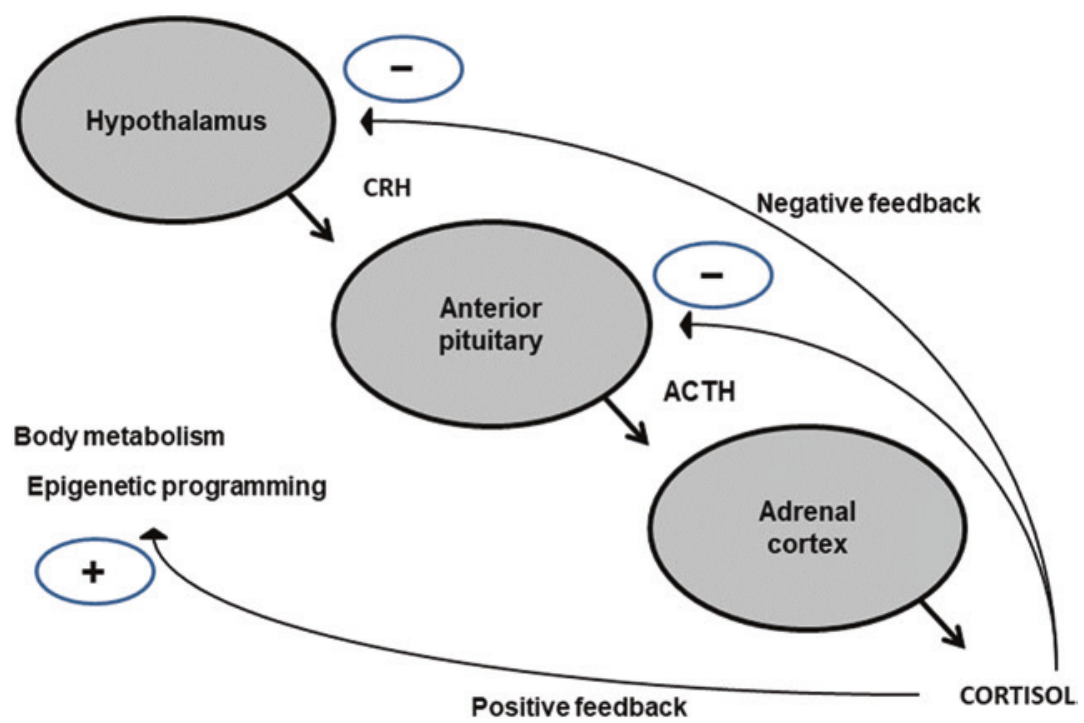

HPA Axis

Fig. 8.1 The HPA axis. Dysregulation of the HPA axis can occur on any level of the system. $\mathrm{ACTH}=$ adrenocorticotropic hormone. $\mathrm{CRH}=$ corticotropin-releasing hormone (๑ Kasia Kozlowska 2019) 
primevally, before the plant and animal kingdoms separated from each other in evolutionary history (Kushiro et al. 2003). Within the body, hormones typically work relatively slowly-in a matter of minutes to hours.

\section{Shifting Gear to Systems Thinking: A Few Tips to the Reader}

The HPA system is immensely complex, and many imminent scientists have dedicated their lives to studying it (see Online Supplement 1.2). By the same token, clinicians from non-medical backgrounds may find this chapter challenging. While we do not expect the reader to master the science of the HPA axis (and we do not make any attempt to present it in any detail here), it is helpful to understand the fundamental processes and how they can go wrong.

In this context, it may be helpful for the reader to shift gear from linear to systems thinking, and to conceptualize the HPA system as having wide-ranging effects throughout the body, all the way down to the level of the individual cell. To capture this complexity, scientists use numerous expressions (e.g., signalling cascades) to communicate that HPA-axis activity cascades across, and affects, numerous body systems.

A key message that we want to communicate in this chapter is that stress-physical or psychological-contributes to the activation or dysregulation of the HPA axis. For most children (including adolescents), the effects of HPA-axis activation in the context of stress are immediate but temporary, with an appropriate short-term increase in available energy in the body. But for children with a history of stress that is chronic, uncontrollable, unpredictable, cumulative, recurrent, or overwhelming, the stress may dysregulate the HPA axis, with the consequence that healthy function is lost. For these children, the HPA axis may activate too much (pattern of overactivation) or too little (pattern of underactivation), or it may reprogram how the brain and other body systems (including the HPA axis itself) respond to stress encountered later in life. These various forms of HPA dysfunction contribute more 
broadly to the dysregulation of the stress system and the dysregulation of the body as a whole-conditions that set the stage for the emergence of functional somatic symptoms.

\section{Cortisol/Glucocorticoids in Energy Regulation}

The end product of activating the HPA axis is cortisol, a water-insoluble hormone that is the most important human glucocorticoid (when discussing the HPA axis in humans, the terms cortisol and glucocorticoid are often used interchangeably). Cortisol produced by the HPA axis-in the adrenal cortex (see Fig. 8.1) —is released in pulses, acting as a messenger that communicates through protein receptors (glucocorticoid receptors) that are present in every cell of the body, including in DNA (deoxyribonucleic acid) and mitochondria. DNA is the cell's genetic material, found in chromosomes within the cell nucleus and in the mitochondria. Genes are defined segments of DNA. Inside each cell, the mitochondria function as energy-producing machines; they regulate and produce energy for the cell and are, as such, a vital part of the body's energy-regulation system.

\section{Patterns of HPA Dysregulation and the Role of Attachment Figures as Psychobiological Regulators}

Dysregulation of the HPA axis can occur on one or more levels of the system (see Fig. 8.1). It may include a pattern characterized by hyper-activity and hyper-responsiveness (higher cortisol levels; enhanced cortisol awakening response; increased adrenocorticotropic hormone $[\mathrm{ACTH}]$; insensitive negative glucocorticoid feedback of the HPA-axis loop; and increased cortisol responses to psychosocial stress or endocrine challenges). Alternately, it may include a pattern characterized by hypo-activity (lower cortisol levels; attenuated diurnal variation of cortisol; enhanced negative feedback within the HPA-axis loop; changed glucocorticoid-receptor sensitivity to cortisol; 
and blunted HPA-axis responsiveness). Mounting evidence suggests that the age of exposure to stress may determine the pattern of HPA dysregulation (Agorastos et al. 2019) (see below). Studies from the maltreatment literature also suggest that the pattern of dysregulation can change from hyper-active to hypo-active during development (see Online Supplement 8.1).

The HPA axis is very sensitive to stress in early childhood. Children who experience significant stress early in development are likely to show a pattern of hyper-activation. Subsequently, as the child ages, the HPA axis may stay hyper-active or may transition to a hypo-active pattern (see Online Supplement 8.1). Importantly, because attachment figures function as biopsychosocial regulators, they are able to buffer HPA-axis activation through sensitive caregiving behaviours (Hostinar et al. 2014; Bernard et al. 2015). In this way, even though stressful events may take place in the life of the child and family, attachment figures can help prevent the child's HPA axis from becoming too activated, thereby buffering the child from the long-term effects of early-life stress. For the neurobiology of nurturing caregiver behaviours, see Online Supplement 8.2, which describes how licking behaviour by mother rats helps buffer HPA-axis activation in rat pups (and which also provides, in effect, a useful model for what happens in humans).

The HPA axis continues to be very sensitive to stress in adolescence. During adolescence the HPA-axis response to stress is more variable, however, and both hyper- and hypo-active patterns are common. Unfortunately, it seems that during adolescence, attachment figures are less able to buffer the HPA-axis response than they are in early childhood (Hostinar et al. 2015).

\section{Cortisol/Glucocorticoids in Epigenetics (Gene Expression) and Plasticity Changes}

In addition to mobilizing energy resources throughout the body, cortisol coordinates plasticity changes - via changes in gene expression-to help the brain and body adapt to stress during the individual's lifetime and across generations. For a brief summary of Michael Meaney's 
groundbreaking research pertaining to glucocorticoids, including changes in gene expression for the glucocorticoid receptor gene in baby rat pups, see Online Supplement 8.2.

Plasticity changes via cortisol's genomic mechanism can be adaptive or maladaptive. For example, regular exercise-a moderate form of pleasurable stress that temporarily increases blood cortisol levels-leads neurons in the hippocampus (a brain region involved in memory and learning) to grow more dendrites and to create more synapses, thereby increasing the size of the hippocampus and improving memory and learning (Erickson et al. 2011; Saraulli et al. 2017). In this way the inclusion of sport in school curricula is helpful for learning.

Another example is stress in utero or early childhood. In this scenario, changes in gene expression-coordinated by cortisol-function to reprogram the manner in which the brain and other tissues in the body (including the HPA axis itself) will respond to stress in the future. The HPA axis thus plays an important role in somatic memory (van der Kolk 1994): significant or cumulative stress in early childhood or adolescence can reprogram the way that the child's brain and body respond to future stress across the child's lifetime (Agorastos et al. 2019). The implication for children with functional somatic symptoms is that HPA-axis activation by past stress may change and intensify the way that each child's stress system responds to current and future stress, unrelated to circulating levels of cortisol at the time of that subsequent stress. This reprogramming can sometime be adaptive, as in cases where the more intensive response helps the child survive in the context of ongoing threat and danger. But it can be maladaptive in safe contexts, where even minor stress would potentially activate an overly robust, inappropriate stress response. In the group vignette below, we see reprogramming of the HPA axis in the context of severe trauma.

During the World Trade Center collapse on September 11, some women directly exposed to the terrorist attack were pregnant. Some of these women went on to develop post-traumatic stress disorder (PTSD), and some did not. When the babies reached one year of age, Rachel Yehuda and colleagues (2005) collected salivary cortisol samples in the babies and the mothers at waking up in the morning and at bedtime. Babies and 
mothers from the mother-with-PTSD group showed lower cortisol levels (a hypo-activity pattern). The lowest cortisol levels were found in babies who were in their third trimester at the time of the collapse. Reduced cortisol levels are linked to an increased vulnerability to PTSD in the face of future stress, and PTSD is associated with functional somatic symptoms. In this way, in the context of future stress, the babies with low cortisol have an increased risk of developing functional somatic symptoms (potentially those associated with a hypo-activity pattern [see below]), PTSD, or other stress-related disorders. For further reading see Yehuda and colleagues (2005) and Gupta (2013).

In the next group vignette, drawn from multiple studies, we see how the HPA axis can be reprogrammed to a hyper-activity pattern by stress.

Girls who are sexually abused show a hyper-activity pattern-overactivation of the HPA axis and too much cortisol-in the aftermath of the trauma. Across the lifespan, they also have higher rates of functional somatic symptoms, including pain, headache, stomachache, nausea, vomiting, eye problems, skin problems, dizziness, fatigue, and non-epileptic seizures. At some point in adulthood, the HPA axis normalizes and then shifts to a hypo-activity pattern-underactivation of the HPA axis and too little cortisol (Trickett et al. 2010). In adulthood the girls have higher rates of functional somatic symptoms-nonspecific chronic pain, functional gastrointestinal disorders, and chronic pelvic pain. Alongside HPA-axis dysregulation, sexually abused girls also show progressive activation, through adulthood, of both the autonomic nervous system and the immune-inflammatory system (for further discussion and additional references concerning this vignette, see Online Supplement 8.1).

In the next group vignette-also from a research study—we describe how psychological stress from adverse life events can reprogram the HPA axis, with the consequence that the individual is less able to fight off infection (in this case the common cold). Most readers will recognize themselves in this vignette because of the common knowledge that our susceptibility to viral infections increases in the context of life stress.

Activation of the HPA axis when the body is fighting infection helps to contain the immune-inflammatory response and keeps it from becoming 
too intense or prolonged. A study by Sheldon Cohen and colleagues (2012) looked at how healthy adults who had experienced recent major life stress (in the last year) responded to inoculation with the common cold virus, versus those who had not experienced such stress. The former group had a greater immune-inflammatory response-and symptoms and signs of illness (of the common cold). The explanation was the HPA axis, hypo-active in the wake of the recent stress, had been unable to appropriately dampen the immune-inflammatory response, with the consequence that the immune-inflammatory response was excessive: it rose to a level needed to combat a much more severe infection. Psychological stress had reprogrammed the HPA axis into a pattern of hypo-activity. For further reading see Cohen and colleagues (2012).

Farther along the maladaptive continuum, extreme, chronic, uncontrollable stress early in life can result in persistently high levels of cortisol and continually excessive energy use-called oxidative stresscausing, for example, dendrites in the vulnerable brain regions to shrink and to thereby impair function (McEwen et al. 2015). Stress-related decreases in brain volume in the hippocampus and brain regions that are part of the brain stress systems have been found in adults with PTSD and children with a history of physical or sexual abuse (for references see Online Supplement 1.3).

In these ways, even though the HPA axis may look like a simple system acting through hormone signals (see Fig. 8.1), it is actually a complicated system that, via glucocorticoid (cortisol) receptors, affects cell function and gene expression across the body.

\section{HPA Dysregulation and Studies of Children with Functional Somatic Symptoms}

Research pertaining to HPA-axis dysregulation in children with functional somatic symptoms is still in the early stages (for references and a summary of studies, see Online Supplement 8.1). We know, however, that in children, recurrent abdominal pain, non-neurological functional somatic symptoms (overtiredness, dizziness, headache, stomach pain, 
vomiting, nausea, and musculoskeletal pain [in the back, neck, shoulders, arms, or legs]), and chronic fatigue syndrome, as well as comorbid conditions such as PTSD and atypical depression, are associated with decreased cortisol levels and HPA-axis hypo-activation. From the adult literature we also know that non-epileptic seizures_-as well as common comorbid conditions such as panic disorder and depression-are associated with increased cortisol levels and HPA-axis hyper-activation. Both patterns of HPA dysregulation are often accompanied by activation or dysregulation in other components of the stress system-in particular, the autonomic nervous system (see Chapter 6) and immune-inflammatory system (Agorastos et al. 2019) (see Chapter 9).

\section{The Take-Home Messages About the HPA Axis for the Mental Health Clinician}

Three key messages pertaining to the HPA axis are important to remember from a clinical perspective.

First, the HPA-axis profile, whether it is hyper- or hypo-active, may affect the child's symptom profile at presentation. For example, because HPA-axis activation suppresses sleep, a hyper-active HPA axis will contribute to sleep arousals and poor sleep (Chrousos et al. 2016) (see also Chapter 5). Alternatively, because cortisol is needed for release of energy resources, hypo-activation of the HPA axis may contribute to symptoms of tiredness and fatigue (Janssens et al. 2012).

Second, loss of synchrony within the HPA axis, between the HPA axis and other components of the stress system, or between the HPA axis and the circadian clock will also affect the child's subjective sense of vitality and well-being because body systems have lost the normal rhythms associated with wellness and energy regulation during the circadian cycle (see also Chapters 4 and 5).

Third, stress-related dysregulation of the HPA axis (as may be present in a child's hyper- or, over time, hypo-activation to stress) is likely to be associated with non-normative responses to future stress and with experience-dependent changes in the brain and body tissues. These 
experience-dependent changes may make the child vulnerable to aberrant responses that manifest as functional somatic symptoms in the context of future stress. In the following vignette of Bellynda, the reader can see how these processes may come into play-in clinical practicein the case of an individual child.

Bellynda was an 11-year-old girl who presented with multiple somatic symptoms - tinnitus and hearing problems, dizziness, blurred vision, nausea, pain in the neck and back, abnormal gait and lack of coordination in the legs, and tremor and flapping of the hands-following three severe episodes of allergic rhinitis (allergic reaction in the nose) and a sudden growth spurt. Bellynda lived with her mother and had no contact with her father. From the family assessment interview, it was clear that Bellynda's symptoms had been triggered by activation of the immune-inflammatory system (via the allergic rhinitis) and dysregulation of the autonomic system (the autonomic system sometimes takes time to adjust following a growth spurt). Bellynda's standing test showed a stable blood pressure and a heart rate increase of 64 (from 80 to 144) beats per minute on standing, confirming the autonomic dysregulation (orthostatic intolerance; see Chapter 6). Her hyperventilation challenge showed chronic hyperventilation (activation of the respiratory motor system alongside the sympathetic system) (see Chapter 7). What was unusual was that apart from this physical stress, Bellynda's recent history was unremarkable: she did not report any other emotional or physical stress. She had, however, experienced significant early-life events in the perinatal period. First, Bellynda's mother had suffered from thyroid cancer and had been operated on during the third trimester of the pregnancy. After the operation Bellynda's mother had been given high doses of thyroxine hormone. Bellynda's mother remembered that she had been unable to sleep with the hormone and that she had felt baby Bellynda kicking and moving inside her in an unsettled way. Because thyroxine hormone is known to up-regulate the stress system, the therapist hypothesized that Bellynda's stress system may have been programmed-in utero-to respond to future stress in an excessively robust way, putting her at risk of developing functional somatic symptoms in response to minor stress. In addition to the exposure to high-dose thyroxine, Bellynda was separated from her mother at four weeks of age, when her mother received radioactive treatment for the thyroid cancer. The protective factor was that Bellynda was 
looked after by her grandmother, who, as an attachment figure, would have buffered the impact of the separation. Finally, the home environment was characterized by high levels of stress during Bellynda's first year of life; her parents separated when she was 11 months old. It is reasonable to hypothesize that the stress associated with these family events also affected the programming, sensitivity, and reactivity of Bellynda's stress system.

As we have seen in this chapter, stress-physical or psychologicalcontributes to activation or dysregulation of the HPA axis. For most children the effects of HPA-axis activation in the context of stress are immediate, with an appropriate increase in available energy in the body. That is exactly what is supposed to happen. But for children with a history of stress that is severe along any of various continuums, the pattern of activation of the entire stress system can be affected, with earlier stress leading to changes in how the body responds to stress encountered later. The exact pattern of HPA-axis activation or dysregulation varies across development and from individual to individual: it depends on both the quality of the stress and its timing. The advent of new technologies - including studies on a group level of analysis- has allowed researchers to begin to identify some of the neurobiological processes that underpin the contribution of HPA-axis activation and dysregulation to functional somatic symptoms. But much is still to be learnt. In Chapter 9, we look at another component of the stress system-the immune-inflammatory system-that is closely interconnected with the autonomic nervous system and HPA axis, and that is also involved in the production of functional somatic symptoms.

\section{References}

Agorastos, A., Pervanidou, P., Chrousos, G. P., \& Baker, D. G. (2019). Developmental Trajectories of Early Life Stress and Trauma: A Narrative Review on Neurobiological Aspects Beyond Stress System Dysregulation. Frontiers in Psychiatry, 10, 118. 
Bernard, K., Hostinar, C. E., \& Dozier, M. (2015). Intervention Effects on

Diurnal Cortisol Rhythms of Child Protective Services-Referred Infants in Early Childhood: Preschool Follow-Up Results of a Randomized Clinical Trial. JAMA Pediatrics, 169, 112-119.

Chrousos, G., Vgontzas, A. N., \& Kritikou, I. (2016). HPA Axis and Sleep. In K. R. Feingold, B. Anawalt, A. Boyce, G. Chrousos, K. Dungan, A. Grossman, J. M. Hershman, G. Kaltsas, C. Koch, P. Kopp, M. Korbonits, R. McLachlan, J. E. Morley, M. New, L. Perreault, J. Purnell, R. Rebar, F. Singer, D. L. Trence, A.Vinik, \& D. P. Wilson (Eds.), Endotext: Comprehensive Free Online Endocrinology Book. South Dartmouth, MA: MDText.com. https://www.ncbi.nlm.nih.gov/books/NBK279071/.

Cohen, S., Janicki-Deverts, D., Doyle, W. J., Miller, G. E., Frank, E., Rabin, B. S., et al. (2012). Chronic Stress, Glucocorticoid Receptor Resistance, Inflammation, and Disease Risk. Proceedings of the National Academy of Sciences of the United States of America, 109, 5995-5999.

Erickson, K. I., Voss, M. W., Prakash, R. S., Basak, C., Szabo, A., Chaddock, L., et al. (2011). Exercise Training Increases Size of Hippocampus and Improves Memory. Proceedings of the National Academy of Sciences of the United States of America, 108, 3017-3022.

Gupta, M. A. (2013). Review of Somatic Symptoms in Post-traumatic Stress Disorder. International Review of Psychiatry, 25, 86-99.

Hostinar, C. E., Johnson, A. E., \& Gunnar, M. R. (2015). Parent Support Is Less Effective in Buffering Cortisol Stress Reactivity for Adolescents Compared to Children. Developmental Science, 18, 281-297.

Hostinar, C. E., Sullivan, R. M., \& Gunnar, M. R. (2014). Psychobiological Mechanisms Underlying the Social Buffering of the HypothalamicPituitary-Adrenocortical Axis: A Review of Animal Models and Human Studies Across Development. Psychological Bulletin, 140, 256-282.

Janssens, K. A., Oldehinkel, A. J., Verhulst, F. C., Hunfeld, J. A., Ormel, J., \& Rosmalen, J. G. (2012). Symptom-Specific Associations Between Low Cortisol Responses and Functional Somatic Symptoms: The Trails Study. Psychoneuroendocrinology, 37, 332-340.

Kleckner, I. R., Zhang, J., Touroutoglou, A., Chanes, L., Xia, C., Simmons, W. K., et al. (2017). Evidence for a Large-Scale Brain System Supporting Allostasis and Interoception in Humans. Nature Human Behaviour, 1, 0069. Kushiro, T., Nambara, E., \& McCourt, P. (2003). Hormone Evolution: The Key to Signalling. Nature, 422, 122. 
McEwen, B. S., Gray, J. D., \& Nasca, C. (2015). 60 Years of Neuroendocrinology: Redefining Neuroendocrinology: Stress, Sex and Cognitive and Emotional Regulation. Journal of Endocrinology, 226, T67-T83.

Picard, M., McEwen, B. S., Epel, E. S., \& Sandi, C. (2018). An Energetic View of Stress: Focus on Mitochondria. Frontiers in Neuroendocrinology, 49, 72-85. Saraulli, D., Costanzi, M., Mastrorilli, V., \& Farioli-Vecchioli, S. (2017). The Long Run: Neuroprotective Effects of Physical Exercise on Adult Neurogenesis from Youth to Old Age. Current Neuropharmacology, 15, 519-533.

Trickett, P. K., Noll, J. G., Susman, E. J., Shenk, C. E., \& Putnam, F. W. (2010). Attenuation of Cortisol Across Development for Victims of Sexual Abuse. Development and Psychopathology, 22, 165-175.

Van Der Kolk, B. A. (1994). The Body Keeps the Score: Memory and the Evolving Psychobiology of Posttraumatic Stress. Harvard Review of Psychiatry, 1, 253-265.

Yehuda, R., Engel, S. M., Brand, S. R., Seckl, J., Marcus, S. M., \& Berkowitz, G. S. (2005). Transgenerational Effects of Posttraumatic Stress Disorder in Babies of Mothers Exposed to the World Trade Center Attacks During Pregnancy. Journal of Clinical Endocrinology and Metabolism, 90, 4115-4118.

Open Access This chapter is licensed under the terms of the Creative Commons Attribution-NonCommercial-NoDerivatives 4.0 International License (http://creativecommons.org/licenses/by-nc-nd/4.0/), which permits any noncommercial use, sharing, distribution and reproduction in any medium or format, as long as you give appropriate credit to the original author(s) and the source, provide a link to the Creative Commons license and indicate if you modified the licensed material. You do not have permission under this license to share adapted material derived from this chapter or parts of it.

The images or other third party material in this chapter are included in the chapter's Creative Commons license, unless indicated otherwise in a credit line to the material. If material is not included in the chapter's Creative Commons license and your intended use is not permitted by statutory regulation or exceeds the permitted use, you will need to obtain permission directly from the copyright holder.

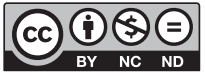

